\title{
Proportional payoffs in legislative bargaining with weighted voting: a characterization
}

\author{
Maria Montero* \\ This is the final version accepted by the Quarterly Journal of \\ Political Science
}

\begin{abstract}
This paper examines the relationship between voting weights and expected equilibrium payoffs in legislative bargaining and provides a necessary and sufficient condition for payoffs to be proportional to weights. This condition has a natural interpretation in terms of the supply and demand for coalition partners. An implication of this condition is that Snyder et al.'s (2005) result, that payoffs are proportional to weights in large replicated games, does not necessarily extend to the smaller games that arise in applications. Departures from proportionality may be substantial and may arise even in well-behaved (homogeneous) games.
\end{abstract}

Keywords: legislative bargaining, weighted voting, proportional payoffs.

\footnotetext{
${ }^{*}$ University of Nottingham, University Park NG7 2RD Nottingham, United Kingdom. e-mail: maria.montero@nottingham.ac.uk. I'm grateful to Jean Derks for MATLAB code for the calculations reported in table 1 .
} 
Many important collective bodies make decisions by weighted majority voting. Examples are the Electoral College in the United States, the International Monetary Fund, the European Union Council of Ministers and any legislature with disciplined political parties. An important question in this setting is how the distribution of votes affects payoffs. Power indices such as the ShapleyShubik index coincide with voting weights only rarely. In contrast, Snyder, Ting and Ansolabehere (2005, p. 982) argue that

Elementary microeconomic theory teaches that in competitive situations perfect substitutes have the same price. In a political setting in which votes might be traded or transferred in the formation of coalitions, one might expect the same logic to apply. If a player has $k$ votes, then that player should command a price for those votes equal to the total price of $k$ players that each have one vote.

Snyder et al. (2005) use a noncooperative bargaining game based on the Baron-Ferejohn (1989) model to show that a voter's expected payoff is proportional to its voting weight. They mention two difficulties in proving this result: corner solutions created by equal recognition probabilities, and nonhomogeneity of the game. Equal recognition probabilities may lead to low-weight voters having disproportionately high payoffs due to proposing power, whereas nonhomogeneous games create a difficulty in that players may be substitutes in some minimal winning coalitions but not in others, and it is not immediately obvious what the competitive price for their votes should be. They address these difficulties by making recognition probabilities proportional to voting weights and by replicating the game a finite (though potentially large) number of times (see Proposition 2 in Snyder et al. (2005)). 
Given that the proof in Snyder et al. (2005) only covers replicated games, how far this result extends to the legislatures with only a few parties that arise in applications is an open question. The present paper provides a necessary and sufficient condition for proportional equilibrium payoffs. This necessary and sufficient condition is relevant for any weighted voting game; the only assumption needed is that recognition probabilities are proportional to the voting weights. This condition can be interpreted in economic terms: there is no excess supply or demand of any player type. An implication of this condition is that, even in the intuitively most favorable case (i.e. uniquely defined homogeneous weights and recognition probabilities proportional to those weights) the equilibrium of the game is not necessarily competitive. It may be possible for larger players to get a disproportionate payoff even if cheaper perfect substitutes appear to be available.

In order to get a rough idea of how often proportional payoffs are predicted in applications, the condition is used to calculate the frequency with which the model actually predicts proportional payoffs in Snyder et al.'s dataset of coalition governments in 14 countries from 1946 to 2001. Proportional payoffs are predicted for about $69 \%$ of the legislatures; this proportion varies between countries and can be as high as 100\% (for Australia and Austria) or as low as $28 \%$ (for Italy). The difference between equilibrium and proportional payoffs may be substantial, both quantitatively and qualitatively, and this is illustrated with some examples from the dataset. Perhaps the most important qualitative deviation is that it is possible for asymmetric parties to have the same equilibrium expected payoff, even though one of the parties is a more desirable coalition partner and has a greater probability of being proposer. Also, minimal winning coalitions are not necessarily proposed in equilibrium (surplus coalitions in which the proposer is the only nonpivotal player are also 
possible).

\section{Preliminaries}

\section{Weighted voting games}

$N=\{1, \ldots, n\}$ is the set of players, $S \subseteq N$ represents a generic coalition and $X$ is the set of alternatives. In the legislative bargaining model under consideration, there is a budget of size 1 to be divided and $X=\left\{x \mid x_{i} \geq 0\right.$ for all $i$ and $\left.\sum_{i \in N} x_{i} \leq 1\right\}$ is the set of all possible allocations. Player $i$ 's preferences are described by the utility function $u_{i}(x)=x_{i}$.

The voting game is described by a set of winning coalitions $W$, where a winning coalition is a coalition that can enforce any alternative in $X$. A voting game is proper if a coalition $S$ and its complement $N \backslash S$ cannot both be winning. A voting game is strong if ties are not possible, i.e., $S$ and $N \backslash S$ cannot both be losing. I assume henceforth that the voting game is proper, but not necessarily strong. A minimal winning coalition (MWC) $S$ is a coalition that is just large enough to win, that is, $S$ is winning but no $T \varsubsetneqq S$ is winning.

The voting game is weighted if it is possible to assign a number of votes (weight) $w_{i} \geq 0$ to each player and to set a threshold $q$ such that $S$ is winning if and only if $\sum_{i \in S} w_{i} \geq q$. The combination $\left[q ; w_{1}, \ldots, w_{n}\right]$ is a representation of the voting game. Many representations $\left[q ; w_{1}, \ldots, w_{n}\right]$ are equivalent in that they produce the same set of winning coalitions. A representation $\left[q ; w_{1}, \ldots, w_{n}\right]$ is called homogeneous if all minimal winning coalitions have the same total weight. For example, $[5 ; 4,3,2]$ is not homogeneous because coalition $\{1,2\}$ has a weight of 7 , whereas coalition $\{2,3\}$ has a weight of 5 . A homogeneous representation of the same game is $[2 ; 1,1,1]$. Homogeneous representations are 
preferred because they give a more transparent description of the situation: $[2 ; 1,1,1]$ reflects the fact that all three players are in a symmetric position (i.e., they are perfect substitutes) since any two of them can form a winning coalition. A game that admits a homogeneous representation is a homogeneous game.

Homogeneous voting weights are not necessarily unique. For example, $[5 ; 3,2,2,1]$ and $[7 ; 4,3,3,1]$ are homogeneous representations of the same game. A possible approach to deal with this indeterminacy is to use minimal integer weights (MIWs); this approach has been taken in the empirical literature ${ }^{1}$ (Ansolabehere et al. (2003), Snyder et al. (2005), Cutler et al. (2014)). A representation has minimal integer weights if all $w_{i}$ 's are integer numbers and there is no representation with smaller weights (see Ostmann (1987), Freixas and Molinero (2009)).

\section{The noncooperative model}

The noncooperative model is the Baron-Ferejohn (1989) model with weighted voting. Given a set of players $N$ and an associated set of winning coalitions $W$, bargaining proceeds as follows. Nature randomly selects one of the players to be the proposer, according to a vector $\theta:=\left(\theta_{1}, \ldots, \theta_{n}\right)$ of recognition probabilities, where $\theta_{i} \geq 0$ for all $i \in N$ and $\sum_{i=1}^{n} \theta_{i}=1$. The proposer then proposes a distribution $\left(x_{1}, \ldots, x_{n}\right)$ of a budget, with $x_{j} \geq 0$ for all $j=1, \ldots, n$

\footnotetext{
${ }^{1} \mathrm{~A}$ large body of empirical literature is devoted to testing Gamson's law, which states that ministerial portfolios are allocated proportionally to the seat shares of parties in government irrespective of the voting weights (see Warwick and Druckman (2006)). Cutler et al. (2014) incorporate both seat shares and MIWs in their statistical model, and find that MIWs have a bearing on which parties get into government, whereas portfolio allocation follows Gamson's law.
} 
and $\sum_{j=1}^{n} x_{j} \leq 1$. This proposal is then voted upon. ${ }^{2}$ If the set of voters in favor of the proposal is a winning coalition, the proposal is implemented and the game ends; otherwise the game proceeds to the next round in which the process is repeated. Players share a discount factor $\delta \in(0,1]$.

A (pure) strategy for player $i$ is a sequence $\sigma_{i}=\left(\sigma_{i}^{t}\right)_{t=1}^{\infty}$, where $\sigma_{i}^{t}$, the $t$-th round strategy of player $i$, prescribes:

1. A proposal, denoted by $x$.

2. A response function assigning "yes" or "no" to all possible proposals by the other players.

Players may condition their actions on the history of play; however the literature focuses on equilibria in which they do not condition on any elements of history other than the current proposal, if any. These equilibria are called stationary subgame perfect equilibria (SSPE). ${ }^{3}$ Stationarity requires that players follow the same strategy at every round $t$ regardless of past offers and responses to past offers. An SSPE always exists (Banks and Duggan, 2000). For a fixed $\delta<1$, all SSPE involve immediate agreement (Okada, 1996) and lead to the same expected payoffs (Eraslan and McLennan, 2013). Expected equilibrium payoffs are usually unique even if $\delta=1$; when they are not, it is possible to obtain a unique prediction by taking the limit when $\delta \rightarrow 1$. Calculations for particular numerical examples assume $\delta \rightarrow 1$.

The logic of the Baron-Ferejohn model is simple. Take any stationary strategy combination. Because of stationarity, player $i$ 's expected payoff computed

\footnotetext{
${ }^{2}$ Voters are assumed to vote on the proposal sequentially. This assumption can be replaced by simultaneous voting plus the additional equilibrium refinement that voters always vote as if their vote makes a difference (see Baron and Kalai (1993)).

${ }^{3}$ Baron and Ferejohn (1989) find a severe multiplicity of SPE in their model, and make a case for the stationarity refinement on the grounds of simplicity of the strategies (Baron and Kalai (1993) make this argument more formally).
} 
at the start of any round is the same in all rounds irrespective of history; denote this expected payoff by $v_{i}$. Player $i$ 's expected payoff after a proposal has (just) been rejected ( $i$ 's continuation value) is then equal to $\delta v_{i}$. These continuation values act as prices. It is optimal for player $i$ to accept any proposal that guarantees him at least $\delta v_{i}$ as a responder and to reject all other proposals. ${ }^{4}$ Given that responders follow these cutoff strategies, it is optimal for player $i$ as a proposer to find the cheapest group of players whose votes are enough to form a winning coalition and to offer each of them exactly $\delta v_{j}$. We say that player $i$ proposes coalition $S$ if $i \in S$ and the proposed payoff vector $x$ has $x_{j}=\delta v_{j}$ for $j \in S \backslash\{i\}, x_{i}=1-\sum_{j \in S \backslash\{i\}} \delta v_{j}$ and $x_{j}=0$ for $j \in N \backslash S$. Let $p_{i}(S)$ be the probability that $i$ proposes $S$. Any SSPE involves a vector of players' acceptance thresholds $\left(\delta v_{i}\right)_{i \in N}$ and a vector of proposal probabilities $\left(p_{i}(S)\right)_{S \ni i, i \in N}$ satisfying two conditions (see Okada (1996), theorem 2):

(1) Proposers propose only the cheapest coalitions available given responders' acceptance thresholds, that is, any coalition with $p_{i}(S)>0$ must minimize $\sum_{j \in T \backslash\{i\}} \delta v_{j}$ (or, equivalently, $\sum_{j \in T} v_{j}$ ) subject to the constraint that $T$ is a winning coalition with $T \ni i$.

(2) Responders' acceptance thresholds coincide with their continuation values, or equivalently,

$$
v_{i}=\theta_{i} \sum_{S: S \ni i} p_{i}(S)\left[1-\sum_{j \in S \backslash\{i\}} \delta v_{j}\right]+\left[\sum_{j \in N \backslash\{i\}} \theta_{j} \sum_{S \supseteq\{i, j\}} p_{j}(S)\right] \delta v_{i} .
$$

Intuitively, this bargaining model is competitive because a player with a disproportionately high $v_{i}$ would be overpriced and get few proposals if any, which would make it difficult for the player to have a high $v_{i}$ in the first place.

\footnotetext{
${ }^{4}$ There is little loss of generality in assuming that ties are always solved in favor of acceptance (see Yan (2002), proposition 2; Eraslan and McLennan (2013), Appendix A).
} 


\section{Replicated games}

Given the original weighted majority game $\left[q ; w_{1}, \ldots, w_{n}\right]$, the game with $r$ replications has $r n$ players and a quota $r q$. The weight vector is found by replacing each player $i$ with $r$ copies with weight $w_{i}$.

Snyder et al. (2005) make no claims on how large $r$ needs to be in order to obtain proportional payoffs. Anecdotal evidence suggests that $r$ is often surprisingly small. However, as Laver et al. (2011) point out, the link between a replicated game and the original game may be tenuous. For example, [5; 4, 3, 2] is a symmetric game with three interchangeable players but its replicated game with $r=2,[10 ; 4,4,3,3,2,2]$, has three non-interchangeable types of players. Likewise, $[3 ; 2,1,1]$ is a game in which player 1 belongs to all winning coalitions and therefore has veto power, whereas the corresponding game with $r=2$, $[6 ; 2,2,1,1,1,1]$, has no veto players. Since the properties of replicated games are not always a good guide to the properties of the original game, equilibria of replicated games may be very different as well. The propositions in Snyder et al. (2005) apply to larger, replicated games rather than to the original game. Hence, the predictions they test are not necessarily equilibrium predictions.

\section{Some simple examples of nonproportionality}

In this section I discuss why equilibrium payoffs may deviate from proportionality, using some simple examples.

The simplest examples of deviation from proportionality are games with a veto player, such as $[3 ; 2,1,1]$. The veto player gets everything if $\delta \rightarrow 1$ (Winter, 1996) even though it has only half of the total weight. It is clear that the substitution argument does not bite in this case, since the veto player must be in all coalitions and cannot be replaced by other players. 
Games with veto players are special since the veto player cannot be replaced at all, hence we would not expect proportionality to hold. ${ }^{5}$ However, lack of substitutability is not confined to games with veto players, as the following example illustrates.

Consider the weighted voting game [5; 3, 2, 2, 1], discussed in Montero (2000). This is a homogeneous game (without a unique homogeneous representation), and the weights reported are MIWs. There are two types of MWCs: the large party together with one of the medium-size parties, and the three smaller parties together. Let $v_{[3]}, v_{[2]}$ and $v_{[1]}$ denote the expected equilibrium payoffs for a player with 3, 2 and 1 votes respectively. Since each medium-size party can form a coalition with either the large party or the two smaller parties, one would expect $v_{[3]}=v_{[2]}+v_{[1]}$. However, there is no particular reason to expect $v_{[2]}=2 v_{[1]}$. A player with 2 votes need not command a price equal to that of two players with 1 vote each, since no two players with 1 vote are available to replace the player with 2 votes. ${ }^{6}$

Example 1 Consider the weighted voting game $[5 ; 3,2,2,1]$. Let $\theta=\left(\frac{3}{8}, \frac{2}{8}, \frac{2}{8}, \frac{1}{8}\right)$. It is easy to see that $v \neq\left(\frac{3}{8}, \frac{2}{8}, \frac{2}{8}, \frac{1}{8}\right)$. All SSPE have $v_{[3]}=\frac{5}{14}, v_{[2]}=\frac{4}{14}$ and $v_{[1]}=\frac{1}{14}$.

Proof. See Online Appendix.

Note that equilibrium payoffs are quite different from the MIWs we started from. In particular, the ratios $v_{[3]} / v_{[1]}$ and $v_{[2]} / v_{[1]}$ are 5 and 4 respectively instead of 3 and 2. Intuitively, there is an excess demand for the mediumsize players. There is competition for the medium-size players, since they are

\footnotetext{
${ }^{5}$ Indeed, Snyder et al. (2005) exclude games with veto players from their analysis.

${ }^{6}$ Situations where one player cannot be replaced by smaller players in a MWC are known as games with steps (see Ostmann (1987)).
} 
needed by both the large and the small player, and there is no competition at all for the small player. As a result, the medium-size players receive too many proposals and the other two players do not receive enough proposals to sustain payoffs proportional to $\theta$.

The ambiguity of the perfect substitutes argument (or, equivalently, the lack of uniqueness of the homogeneous representation) is not the only reason why equilibrium payoffs may differ from the MIWs. The following example illustrates the lack of proportionality of equilibrium payoffs in a particularly surprising setting, where this issue does not arise.

Example 2 Consider the game $[20 ; 5,5,5,5,5,1,1,1,1,1,1,1,1,1]$. There are two types of $M W C$ in this game: four of the large players, or three of the large players together with five of the small players. The game is clearly homogeneous; furthermore, it has a unique homogeneous representation (up to a multiplicative constant). The substitutability argument points in a very clear direction: a large player can be replaced by five small players, and should get five times as much. Suppose players are recognized with probabilities proportional to their voting weight, i.e., a large player is recognized with probability $\frac{5}{34}$ and a small player is recognized with probability $\frac{1}{34}$. It turns out that the equilibrium is such that $v_{[5]}=\frac{50}{331}$ and $v_{[1]}=\frac{9}{331}$. Hence, the large players are getting a disproportionately high payoff since $v_{[5]}>5 v_{[1]}$.

Proof. In order to show that this is an equilibrium, we need to find strategies that lead to the expected payoffs and are optimal given the expected payoffs. The strategies are as follows: all players propose a coalition of three large players and five small players, and the proposer offers the coalition partners either $\frac{50}{331}$ (for large players) or $\frac{9}{331}$ (for small players). As a responder, a large player votes in favor of any proposal that gives him at least $\frac{50}{331}$, and a small 
player votes in favor of any proposal that gives him at least $\frac{9}{331}$. Proposers are acting optimally given the responders' prices: no other winning coalition would be cheaper. Responders are acting optimally provided that expected payoffs are indeed those, so it only remains to check that expected payoffs are as assumed given the strategies:

$$
\begin{aligned}
& v_{[5]}=\frac{5}{34}\left[1-2 \times \frac{50}{331}-5 \times \frac{9}{331}\right]+\frac{20}{34} \frac{2}{4} \frac{50}{331}+\frac{9}{34} \frac{3}{5} \frac{50}{331}=\frac{50}{331} \\
& v_{[1]}=\frac{1}{34}\left[1-3 \times \frac{50}{331}-4 \times \frac{9}{331}\right]+\frac{25}{34} \frac{5}{9} \frac{9}{331}+\frac{8}{34} \frac{4}{8} \frac{9}{331}=\frac{9}{331} .
\end{aligned}
$$

It is tempting to conclude that the trouble with the previous example is that there are not enough smaller players to replace the large players. All coalitions that form in equilibrium are of type [55511111]. Proposers would rather replace one of the coalition partners of type [5] with five players of type [1], but this is not possible because there are only nine of those and five are already in the coalition. Indeed, adding another small player would lead to proportional payoffs. Interestingly, this is not the whole story: removing one of the small players would also lead to proportional payoffs (more on this in the next section).

\section{A necessary and sufficient condition for propor- tionality}

\section{Balanced collections of coalitions}

Given a weighted voting game $\left[q ; w_{1}, \ldots, w_{n}\right]$, let $W^{*}$ denote the collection of winning coalitions with minimum total weight, i.e., $W^{*}=\arg \min _{S \in W} \sum_{i \in S} w_{i}$. 
If the prices players charge for their cooperation are proportional to the weights, $W^{*}$ is the set of coalitions that are likely to form since they are the cheapest. But are those prices competitive? Suppose a player $i$ belongs to all coalitions in $W^{*}$, and another player $j$ does not belong to any of them. Clearly, player $i$ is underpriced relative to $j$. More generally, even if all players belong to at least one coalition in $W^{*}$, no player should be systematically overrepresented or underrepresented in the list of cheapest coalitions. This idea is formalized by requiring that $W^{*}$ is a weakly balanced collection of coalitions (the definition of a balanced collection of coalitions goes back to Bondareva (1963); see also Shapley (1967)).

Definition 3 Let $W^{*}$ be the set of winning coalitions with minimum total weight. For each $S \in W^{*}$, let $\lambda_{S} \geq 0$ be a weight assigned to $S$. The set $W^{*}$ is weakly balanced if there is a set of weights $\left(\lambda_{S}\right)_{S \in W^{*}}$ such that for every voter $i \in N$ it is the case that $\sum_{S \ni i} \lambda_{S}=1$.

The property of weak ${ }^{7}$ balancedness can be interpreted as follows. If $W^{*}$ is weakly balanced, we can construct a probability distribution over the coalitions in $W^{*}$ such that all players are equally likely to be in the coalition that forms. This probability distribution is found by renormalizing the weights so that they add up to 1, i.e. $p(S)=\frac{\lambda_{S}}{\sum_{T \in W^{*}} \lambda_{T}}$ for each $S \in W^{*}$.

The collection of coalitions $W^{*}$ is weakly balanced if and only if the following system of equations has a solution. Find $\left(\lambda_{S}\right)_{S \in W^{*}}$ such that

$$
\begin{aligned}
\sum_{S \ni i} \lambda_{S} & =1 \text { for all } i \in N \\
\lambda_{S} & \geq 0 \text { for all } S \in W^{*}
\end{aligned}
$$

I now illustrate the definition with some examples.

\footnotetext{
${ }^{7}$ The word "weak" refers to the fact that $\lambda_{S} \geq 0$ is required rather than $\lambda_{S}>0$.
} 
Example 4 Consider the game $[3 ; 2,1,1,1]$. All MWCs have the same total weight, hence $W^{*}=W^{m}=\{\{1,2\},\{1,3\},\{1,4\},\{2,3,4\}\}$. The relevant system of equations is

$$
\begin{aligned}
\lambda_{\{1,2\}}+\lambda_{\{1,3\}}+\lambda_{\{1,4\}} & =1 \\
\lambda_{\{1,2\}}+\lambda_{\{2,3,4\}} & =1 \\
\lambda_{\{1,3\}}+\lambda_{\{2,3,4\}} & =1 \\
\lambda_{\{1,4\}}+\lambda_{\{2,3,4\}} & =1 \\
\lambda_{\{1,2\}}, \lambda_{\{1,3\}}, \lambda_{\{1,4\}}, \lambda_{\{2,3,4\}} & \geq 0
\end{aligned}
$$

The (unique) solution to this system is $\lambda_{\{1,2\}}=\lambda_{\{1,3\}}=\lambda_{\{1,4\}}=\frac{1}{3}$, $\lambda_{\{2,3,4\}}=\frac{2}{3}$. If we renormalize these values such that they add up to 1 , they can be interpreted as probabilities of the respective coalitions: $p(\{1,2\})=$ $p(\{1,3\})=p(\{1,4\})=\frac{1}{5}$ and $p(\{2,3,4\})=\frac{2}{5}$. Given these probabilities, each of the players is included in the final coalition with probability $\frac{3}{5}$. Player 1 is in more MWCs than other players, but this difference can be compensated by making coalition $\{2,3,4\}$ more likely.

Example 1 on the other hand is a clear case of violation of this condition. Since the game is homogeneous, all MWCs have the same total weight and $\left.W^{*}=W^{m}=\{\{1,2\},\{1,3\},\{2,3,4\})\right\}$. The system is then

$$
\begin{aligned}
\lambda_{\{1,2\}}+\lambda_{\{1,3\}} & =1 \\
\lambda_{\{1,2\}}+\lambda_{\{2,3,4\}} & =1 \\
\lambda_{\{1,3\}}+\lambda_{\{2,3,4\}} & =1 \\
\lambda_{\{2,3,4\}} & =1 \\
\lambda_{\{1,2\}}, \lambda_{\{1,3\}}, \lambda_{\{2,3,4\}} & \geq 0
\end{aligned}
$$

The fourth equation requires $\lambda_{\{2,3,4\}}=1$. Substituting this value into the second and third equations gives $\lambda_{\{1,2\}}=\lambda_{\{1,3\}}=0$, which then contradicts 
the first equation. The system has no solution, hence the set $W^{*}$ is not weakly balanced. Player 4 is an inferior player (Napel and Widgrén, 2001) in that it can only be in a MWC when players 2 and 3 are also present; since player 1 also needs either player 2 or player 3 , it is impossible for all players to be in the final coalition with equal probability.

Example 2 is a more subtle instance of the same problem. There are two types $^{8}$ of MWC, [5555] and [55511111]. Even in the most favorable case for the small players, which is when the only coalition type that forms is [55511111], it is still the case that a type [5] player ends up in the coalition with probability $\frac{3}{5}$, whereas a type [1] player only ends up in the coalition with probability $\frac{5}{9}<\frac{3}{5}$.

Either adding or removing a player of type [1] to example 2 would make the balancedness property hold. Adding a player would introduce a new type of MWC that favors the small players, [551111111111]. Removing a player leaves the two types of MWC unchanged, but it gives individual type [1] players a greater chance of being part of coalition type [55511111].

\section{The result}

The main result of this paper is that weak balancedness of the set $W^{*}$ is a necessary and sufficient condition for the existence of an equilibrium with proportional payoffs.

Proposition 5 Let $\left[q ; w_{1}, \ldots, w_{n}\right]$ be an arbitrary weighted majority game, normalized so that $\sum_{i \in N} w_{i}=1$, and let $\theta=w$. There exists an SSPE with $v=w$

\footnotetext{
${ }^{8}$ Note that it is sufficient to search for solutions of system (1) such that $\lambda_{S}=\lambda_{S^{\prime}}$ if $S$ and $S^{\prime}$ are of the same type. If a solution to the system exists, a symmetric solution must also exist (given an asymmetric solution, we can construct a symmetric one by setting each $\lambda_{S}$ equal to the average weight of coalitions of that type).
} 
if and only if $W^{*}$ is weakly balanced.

Proof. See next section.

Note that the value of the discount factor $\delta \in(0,1]$ does not affect the necessary and sufficient condition. If the condition is satisfied, an equilibrium with proportional payoffs exists irrespective of $\delta$, and the only effect of impatience is that all continuation values shrink proportionally and the proposer advantage increases. If the condition is not satisfied, expected equilibrium payoffs cannot be proportional for any $\delta>0$, and their actual value does in general depend on $\delta$.

There are no requirements on $[q ; w]$ in order for the condition to apply. Note however that the condition has almost no chance to hold if $w$ is the vector of seat shares, since in general not all parties belong to a coalition with the minimum number of seats. ${ }^{9}$ MIWs on the other hand ensure that all players belong to a winning coalition of minimum total weight, though even in this case the condition does not necessarily hold as we have seen. ${ }^{10}$

In order to check whether the condition holds, one needs to solve a system of $n$ (the number of players) linear equations with $m$ (the number of winning

\footnotetext{
${ }^{9}$ For example, assuming simple majority voting, the German Bundestag as of September 2014 would be associated with the weighted majority game $[316 ; 311,192,64,63]$. There are four minimal winning coalitions: $\{1,2\},\{1,3\},\{1,4\},\{2,3,4\}$. The coalition with the minimum total number of seats is $\{2,3,4\}$, with 319 seats. Party 1 does not belong to any coalition with 319 seats, hence the corresponding $W^{*}$ would not be balanced.

${ }^{10}$ Even though there are no requirements on $[q ; w]$ in order for the condition to apply, there is an important requirement on the game form: recognition probabilities must be proportional to $w$. As Kalandrakis (2006) has shown, recognition probabilities have a strong influence on equilibrium payoffs. Diermeier and Merlo (2004) found some empirical support for the hypothesis of formateur selection being proportional to seat shares. To the best of my knowledge there has been no empirical testing of selection proportional to voting weights.
} 
coalitions of minimum weight) unknowns, with $m$ additional constraints requiring the value of each of the unknowns to be nonnegative. Just writing down the equations can be a lengthy process. For example, one of the games in the database is $[314 ; 116,108,108,98,39,33,29,18,14,13,11,10,6,6,5,4,3,2,1,1,1]$, which has 10,790 MWCs, of which 8,616 are in $W^{*} !^{11}$ As to solving the system, note that (1) looks like a linear programming problem (each equality can be transformed into two inequalities) except that there is no objective function; hence the matter at stake is whether the program is feasible. One can use linear programming methods to answer this question (see Vanderbei, 2008, chapter 2). ${ }^{12}$

Proposition 5 strengthens an earlier result of Montero (2006). Montero (2006) shows that, if $\theta$ coincides with the nucleolus (Schmeidler (1969)), $v$ coincides with the nucleolus as well. A sufficient condition automatically follows from that earlier result: if the weights happen to be proportional to the nucleolus, expected payoffs are proportional to the weights. Peleg (1968) shows that MIWs are proportional to the nucleolus for all strong homogeneous games, hence the game being in this class is a sufficient condition for the proportionality of expected payoffs. The condition in Montero (2006) is sufficient but not necessary. ${ }^{13}$ The condition in proposition 5 is both necessary and sufficient,

\footnotetext{
${ }^{11}$ I am grateful to Jean Derks for providing MATLAB code that automatically generates the equations taking $(q, w)$ as an input.

${ }^{12} \mathrm{~A}$ more roundabout way of checking the condition involves solving a related linear programming problem and comparing the optimal value of the objective function with $1-$ $\min _{S \in W} \sum_{i \in S} w_{i}$. This method is based on Peleg and Rosenmüller (1992) and Derks and Kuipers (1997), and is discussed in the online appendix.

${ }^{13}$ For example, the nucleolus of the game $[10 ; 3,3,3,2,2,2]$ is $\left(\frac{2}{9}, \frac{2}{9}, \frac{2}{9}, \frac{1}{9}, \frac{1}{9}, \frac{1}{9}\right)$, which is not a system of weights at all, and nevertheless expected payoffs are proportional to the MIWs. I'm grateful to Peter Sudhölter for pointing out this example, which appears in Kopelowitz (1967).
} 
as shown in the next section.

It is worth noting that the condition does not have a straightforward connection with other properties such as the homogeneity of the game. The condition always holds for strong homogeneous games, but may hold for other games as well. For example, it holds for the game [30;14, 14, 12, 4, 4, 4, 4, 1, 1], which is neither homogeneous nor strong. ${ }^{14}$

\section{Relation to repeated bargaining}

A very substantial literature analyzes repeated bargaining with dynamic linkages between different bargaining games. The most common assumption in this literature is that the agreement reached in one stage game becomes the status quo for the next stage game (see Kalandrakis (2004)). Even though the motivation of the present paper is very different, proposition 5 has some implications for repeated bargaining where the dynamic linkage works through the recognition probabilities. Specifically, suppose players play the bargaining game repeatedly, with the status quo remaining at 0 and the voting weights and quota remaining constant, but with an endogenous recognition rule such that each player's recognition probability equals its expected equilibrium payoff (rather than its realized payoff as in Jeon (2015)) from the previous game. Suppose furthermore that players are farsighted within a game but do not take into account that an agreement reached in the current game affects the recognition probabilities in the next one. Under these assumptions, proposition 5 can be modified to obtain a characterization of the set of interior fixed points

\footnotetext{
${ }^{14}$ Putting Proposition 5 together with Proposition 2 in Snyder et al. (2005), it follows that the condition must be satisfied for sufficiently large replica games. Kurz et al. (2014) show a stronger result: weights of large replica games coincide with the nucleolus.
} 
of this process. ${ }^{15}$ The price to pay for the adaptation of proposition 5 to an arbitrary payoff vector is that a) the characterization only applies to payoff vectors $x$ with $x_{i}>0$ for all $i$, while the result on weights allows $w_{i}=0$, which is an important case since parties with a positive number of seats may nevertheless have a 0 voting weight in a minimal integer representation; and b) the proof is slightly more lengthy. To state the result formally, denote the set of winning coalitions with minimum $\sum_{i \in S} x_{i}$ as $\mathcal{W}^{*}(x)$. This set generalizes the set $W^{*}$, which would be $\mathcal{W}^{*}(w)$ in this notation.

Corollary 6 Let $\left[q ; w_{1}, \ldots, w_{n}\right]$ be an arbitrary weighted majority game, and $x$ be a payoff vector (not necessarily a set of weights for the game) such that $x_{i}>0$ for all $i \in N$ and $\sum_{i \in N} x_{i}=1$. Let $\theta=x$. There exists an SSPE with $v=x$ if and only if $\mathcal{W}^{*}(x)$ is weakly balanced.

Proof. See Online Appendix.

\section{Proof of proposition 5}

Because there are no restrictions on $\left[q ; w_{1}, \ldots, w_{n}\right]$, it is possible that no coalition has exactly $q$ votes. Let $\min _{S \in W} \sum_{i \in S} w_{i}:=\bar{q} \cdot{ }^{16}$

1. Necessity. Suppose we have an SSPE with $v=w$. Expected payoffs are given by

$$
v_{i}=\theta_{i} \sum_{S: S \ni i} p_{i}(S)\left[1-\sum_{j \in S \backslash\{i\}} \delta v_{j}\right]+r_{i} \delta v_{i}
$$

where $v_{i}$ is $i$ 's expected payoff, $\theta_{i}$ is the probability that $i$ is selected to be proposer, $p_{i}(S)$ is the probability that $i$ proposes $S$ conditional on $i$ being

\footnotetext{
${ }^{15} \mathrm{I}$ 'm grateful to an anonymous referee for pointing this out.

${ }^{16}$ For example, if $w=\left(\frac{5}{13}, \frac{4}{13}, \frac{4}{13}\right)$ and $q=\frac{7}{13}$, there is no coalition with exactly $\frac{7}{13}$ votes and $\bar{q}=\frac{8}{13}$.
} 
the proposer, and $r_{i}$ is the probability that $i$ receives a proposal from another player.

Consider first the case in which $w_{i}>0$ for all $i$ and each player belongs to at least one coalition in $W^{*}$. Then, if expected payoffs coincide with $w$, the optimal coalitions for the proposer are the coalitions in $W^{*}$ to which it belongs. Since these coalitions have a total weight of $\bar{q}, \sum_{j \in S \backslash\{i\}} v_{j}=\bar{q}-w_{i}$ for all the proposed coalitions, and $\sum_{S: S \ni i} p_{i}(S)\left[1-\sum_{j \in S \backslash\{i\}} \delta v_{j}\right]$ can be written as $1-\delta\left(\bar{q}-w_{i}\right)$. Since both $v_{i}$ and $\theta_{i}$ coincide with $w_{i}$ for all $i$, we can write the equation for expected payoffs as

$$
w_{i}=w_{i}\left[1-\delta\left(\bar{q}-w_{i}\right)\right]+r_{i} \delta w_{i}
$$

Dividing by $w_{i}$ (which by assumption is positive) and re-arranging terms, we find $\delta\left(\bar{q}-w_{i}\right)=\delta r_{i}$, which implies $\bar{q}=r_{i}+w_{i}$ since $\delta>0$. Given that $w_{i}$ is also the probability of being proposer, we see that the total probability of being part of the final coalition (the probability of being proposer, $w_{i}$, plus the probability of being responder, $r_{i}$ ) must be the same for all players. This implies that, if $p(S)$ is the equilibrium probability of coalition $S$ forming, $\sum_{S \ni i} p(S)=\bar{q}$ for all $i$. Notice also that only coalitions with $\bar{q}$ votes form in equilibrium (other coalitions are too expensive), so we may write $\sum_{S: S \in W^{*}, S \ni i} p(S)=\bar{q}$ for all $i$. If we divide both sides of the equation by $\bar{q}$ and define $\lambda_{S}:=p(S) / \bar{q}$, we obtain $\sum_{S: S \in W^{*}, S \ni i} \lambda_{S}=1$ for all $i$. In other words, the set of minimal winning coalitions with $\bar{q}$ votes must be weakly balanced.

If there is a player with $w_{i}>0$ who does not belong to any of the coalitions with exactly $\bar{q}$ votes, this player needs to buy more than $\bar{q}-w_{i}$ votes, and its payoff as a proposer is less than $1-\delta\left(\bar{q}-w_{i}\right)$. We may then write

$$
v_{i}<\theta_{i}\left[1-\delta\left(\bar{q}-w_{i}\right)\right]+r_{i} \delta v_{i} .
$$


If we replace $v_{i}$ and $\theta_{i}$ by $w_{i}$ and divide everything by $w_{i}$, collecting terms we find $r_{i}+w_{i}>\bar{q}$. Since $\bar{q}>\frac{1}{2}$ we find that $r_{i}+w_{i}>\frac{1}{2}$, i.e., player $i$ 's probability of being in the final coalition is above $\frac{1}{2}$. Let $S$ be one of the coalitions with $\bar{q}$ votes. Players in $S$ never include $i$ in their proposal. But this then implies that player $i$ is in the final coalition with a probability of at most $1-\bar{q}$, which is less than $\frac{1}{2}$. Hence, there cannot be an equilibrium with $v=w$ in which a player does not belong to any coalition in $W^{*}$.

If $v=w$ and $w_{i}=0$ for some $i$, we can still show that the set $W^{*}$ must be weakly balanced. Note that players with $w_{i}=0$ trivially belong to at least one coalition in $W^{*}$. If $w_{i}=0$, the coalition that forms can be viewed as including $i$ (since $i$ receives $\delta v_{i}$ ) or excluding $i$ (since $i$ receives 0 ). Choose a player $k$ with $w_{k}>0$ and adopt the arbitrary accounting convention that players with $w_{i}=0$ are considered part of the coalition if and only if player $k$ is part of the coalition $^{17}$. It follows from the analysis above that $\sum_{S \in W^{*}, S \ni i} \lambda_{S}=1$ for all $j$.

2. Sufficiency. Suppose $W^{*}$ is weakly balanced. As in Montero (2006), we can use the weights $\lambda_{S}$ to construct a mixed strategy equilibrium in which $v=w$. As a proposer, player $i$ proposes one of the coalitions in $W^{*}$ to which it belongs according to the probability distribution $p_{i}(S)=\lambda_{S}$ for all $S$ such that $S \in W^{*}, S \ni i ; p_{i}(S)=0$ for all other $S$. Proposing $S$ means that player $i$ sets $x_{j}=\delta w_{j}$ for all $j \in S \backslash\{i\}, x_{i}=1-\sum_{j \in S \backslash\{i\}} \delta w_{j}$ and $x_{j}=0$ for all $j \in N \backslash S$. Since by assumption $\sum_{S \ni i} \lambda_{S}=1$ for all $i$, the strategy is well defined. As a responder, player $i$ accepts proposals if and only if $x_{i} \geq \delta w_{i}$.

I now show that this strategy combination leads to $v_{i}=w_{i}$ for all $i$. This

\footnotetext{
${ }^{17}$ The assumption that $w$ is a weight vector for the game rather than an arbitrary payoff vector plays an important role here. It is because $w_{i}$ is both $i$ 's payoff and $i$ 's weight that we can add or remove player $i$ at will from coalitions in $W^{*}$ and still get a coalition in $W^{*}$. If $w$ is not a vector of weights, removing $i$ from a coalition may produce a losing coalition, i.e., a coalition outside $W^{*}$.
} 
is trivially the case if $w_{i}=0$, since by assumption this player has no chance of being proposer and no other player offers $i$ a positive payoff as a responder. If $w_{i}>0$, player $i$ 's expected payoff given this strategy combination equals

$$
v_{i}=\theta_{i} \sum_{S: S \ni i} p_{i}(S)\left[1-\sum_{j \in S \backslash\{i\}} \delta w_{j}\right]+\left[\sum_{j \in N \backslash\{i\}} \theta_{j} \sum_{S \supseteq\{i, j\}} p_{j}(S)\right] \delta w_{i} .
$$

By assumption, $\theta_{i}=w_{i}$. Player $i$ 's payoff as a proposer can be written as $1-\delta\left(\bar{q}-w_{i}\right)$ since player $i$ only proposes coalitions in $W^{*}$ and by definition these coalitions have a total weight of $\bar{q}$. The probability of receiving a proposal, $\sum_{j \in N \backslash\{i\}} \theta_{j} \sum_{S \supseteq\{i, j\}} p_{j}(S)$, can be rewritten as $\sum_{S \ni i} \sum_{j \in S \backslash\{i\}} \theta_{j} p_{j}(S)$. Hence,

$$
v_{i}=w_{i}\left[1-\delta\left(\bar{q}-w_{i}\right)\right]+\left[\sum_{S \ni i} \sum_{j \in S \backslash\{i\}} \theta_{j} p_{j}(S)\right] \delta w_{i} .
$$

Since by construction only coalitions in $W^{*}$ are proposed and those have $p_{j}(S)=\lambda_{S}$, we can write

$$
\begin{aligned}
v_{i} & =w_{i}\left[1-\delta\left(\bar{q}-w_{i}\right)\right]+\left[\sum_{S: S \ni i, S \in W^{*}} \sum_{j \in S \backslash\{i\}} \theta_{j} \lambda_{S}\right] \delta w_{i}= \\
& =w_{i}\left[1-\delta\left(\bar{q}-w_{i}\right)\right]+\left[\sum_{S: S \ni i, S \in W^{*}} \lambda_{S} \sum_{j \in S \backslash\{i\}} \theta_{j}\right] \delta w_{i} .
\end{aligned}
$$

Furthermore, since $\theta_{j}=w_{j}$ and $\sum_{j \in S \backslash\{i\}} w_{j}=\bar{q}-w_{i}$ for all $S \in W^{*}$ we have $\sum_{j \in S \backslash\{i\}} \theta_{j}=\bar{q}-w_{i}$. We can then write

$$
\begin{aligned}
v_{i} & =w_{i}\left[1-\delta\left(\bar{q}-w_{i}\right)\right]+\left[\sum_{S: S \ni i, S \in W^{*}} \lambda_{S}\left(\bar{q}-w_{i}\right)\right] \delta w_{i}= \\
& =w_{i}\left[1-\delta\left(\bar{q}-w_{i}\right)\right]+\left[\left(\bar{q}-w_{i}\right) \sum_{S: S \ni i, S \in W^{*}} \lambda_{S}\right] \delta w_{i}=w_{i}
\end{aligned}
$$

where the last equality follows from $\sum_{S: S \ni i, S \in W^{*}} \lambda_{S}=1$. 
Because $v_{i}=w_{i}$ for all $i$, players are behaving optimally both as proposers and as responders. Proposers propose only the cheapest coalitions available given the responders' acceptance thresholds (i.e., coalitions in $W^{*}$ ) and responders accept proposals if and only if $x_{i} \geq \delta v_{i}$. Hence, we have an SSPE.

\section{Predicted deviations from proportionality in ap- plications}

\section{Predicted frequency of the deviations}

Because the condition in Proposition 5 is necessary and sufficient we have a characterization, and are able to answer the question of whether equilibrium payoffs are proportional to the voting weights in any particular case. Table 1 shows the frequency of the proportional equilibrium payoffs prediction for the weight distributions in Snyder et al. (2005)'s dataset. ${ }^{18}$ This frequency provides some guidance as to how likely the condition is to hold in applications. ${ }^{19}$ As a byproduct, it also indicates the proportion of cases in which the predictions tested by Snyder et al. are supported by the equilibrium of their

\footnotetext{
${ }^{18}$ These calculations use the MIWs provided by Snyder et al. in their supplementary material. All frequencies are computed as a fraction of the total number of observations in the dataset, where each government is an observation.

${ }^{19}$ Another potential application is the EU Council of Ministers. The condition fails to hold for most of the historical weight distributions (see Le Breton et al. (2012)).
} 
theoretical model.

Table 1. Frequency of proportional equilibrium prediction

\begin{tabular}{lccc} 
& Observations & Proportional & Frequency \\
\hline Australia & 26 & 26 & 1 \\
Austria & 23 & 23 & 1 \\
Belgium & 36 & 25 & 0.69 \\
Denmark & 32 & 25 & 0.78 \\
Finland & 44 & 19 & 0.43 \\
Iceland & 22 & 18 & 0.82 \\
Ireland & 22 & 12 & 0.55 \\
Italy & 46 & 13 & 0.28 \\
Luxembourg & 17 & 11 & 0.65 \\
Netherlands & 23 & 10 & 0.43 \\
Norway & 27 & 26 & 0.96 \\
Portugal & 15 & 11 & 0.73 \\
Sweden & 25 & 24 & 0.96 \\
(West) Germany & 20 & 19 & 0.95 \\
\hline All countries & 378 & 262 & 0.69
\end{tabular}

Some of these observations correspond to trivial cases in which either one party has the overall majority or all parties are de facto symmetric. If trivial cases (defined as cases in which all minimal integer weights are 0 or 1 ) are excluded, the overall frequency of the proportional equilibrium prediction drops to about $59 \%$. 


\section{Predicted size of the deviations}

The necessary and sufficient condition in Proposition 5 provides a yes/no answer on proportionality: if it fails, expected equilibrium payoffs cannot be proportional. But how far are they from being proportional? The online appendix compares equilibrium payoffs and weights for all games in the dataset with at most 7 players that fail to satisfy the condition (excluding games with a veto player, of which there are two in the database). In this section, I look at the difference between proportional and equilibrium payoffs in two examples from the database, $[9 ; 5,4,4,1,1,1]$ and $[17 ; 9,8,5,4,4,1,1]$.

Figure 1 shows the weights $w_{i}$ (the MIWs, normalized so that they add up to 1) and expected equilibrium payoffs $v_{i}$ for these two games. Each bar represents one player. Most players get an expected payoff that is not too far from their voting weight; for example, player 1 in game $[17 ; 9,8,5,4,4,1,1]$ has an expected payoff of 0.278 (this is also player 1's predicted payoff as a coalition partner) and a normalized voting weight of 0.281 . However, there are also players whose expected payoffs are substantially different from their weight shares, and this is often true for the smallest player type, as the online appendix illustrates. In game $[17 ; 9,8,5,4,4,1,1]$, player 7 has a weight share of 0.031 but an expected payoff of only 0.014. Taking weights rather than expected payoffs as the theoretical prediction for realized allocations matters more or less depending on which players are the coalition partners; in particular, if player 2 is the proposer and player 1 is the coalition partner, the difference is almost imperceptible.

Figure 2 shows $\frac{v_{i}}{w_{i}}$, the ratio of payoffs to weights, for the same two examples. This ratio measures how much of a player's weight is translated into expected equilibrium payoffs; if expected equilibrium payoffs were proportional to weights it would always be 1 . Even though most player types have ratios 
$[9 ; 5,4,4,1,1,1]$

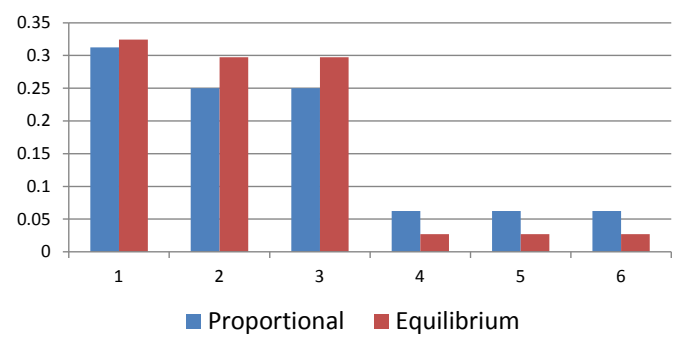

$[17 ; 9,8,5,4,4,1,1]$

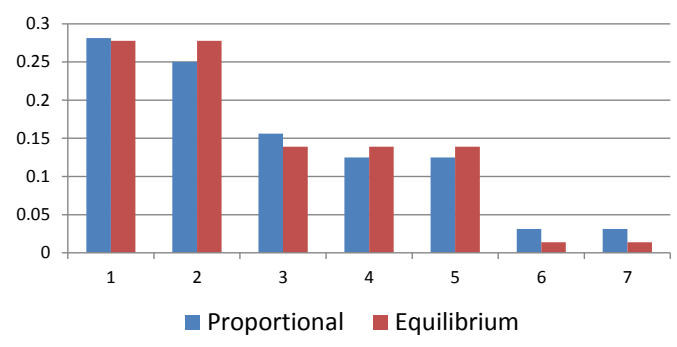

Figure 1: Equilibrium versus proportional payoffs in two examples

$[9 ; 5,4,4,1,1,1]$

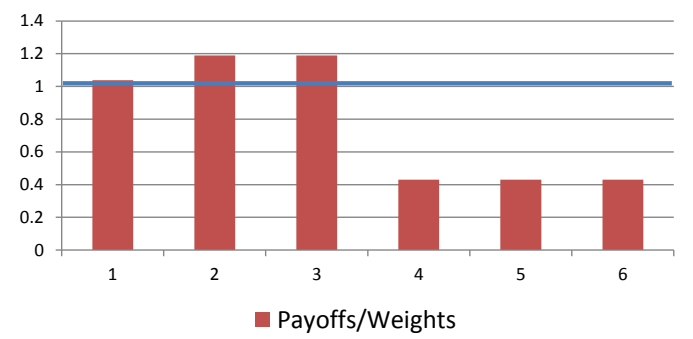

$[17 ; 9,8,5,4,4,1,1]$

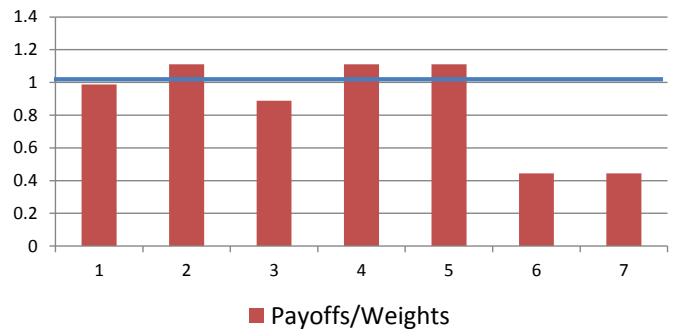

Figure 2: Ratio of payoffs to weights in two examples

close to 1 , the smallest player types get only about $44 \%$ of their weight share in these two examples. The picture can also be interpreted as a comparison of payoffs per vote for different players. The smallest players get a much lower payoff per vote.

A third measure of deviation from proportionality is the relative payoffs $v_{i} / v_{n}$, i.e. the exchange rate between players according to equilibrium predictions. If expected equilibrium payoffs were proportional, this exchange rate would always be equal to $w_{i} / w_{n}$ (in particular, if $w_{n}=1$, this ratio would replicate the MIWs). Because player $n$ often gets very little, the ratios between a player's payoff and the payoff of the smallest player are very different 
$[9 ; 5,4,4,1,1,1]$

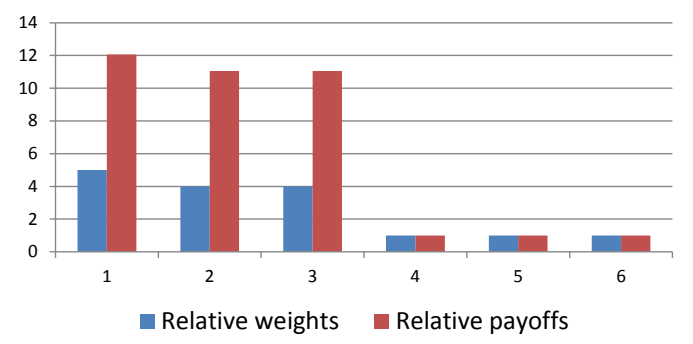

$[17 ; 9,8,5,4,4,1,1]$

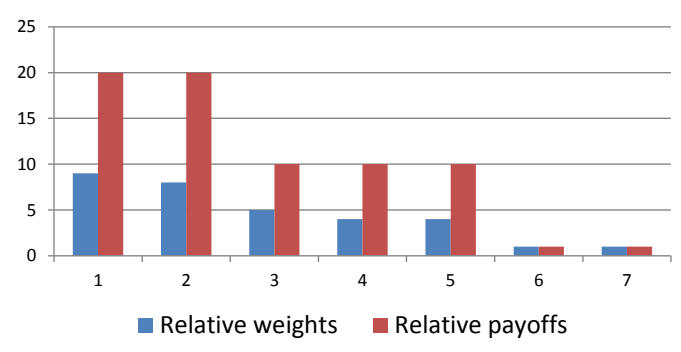

Figure 3: Relative weights versus relative payoffs in two examples

from $w_{i} / w_{n}$. In the game $[9 ; 5,4,4,1,1,1]$, a player with 5 votes does not get 5 times as much as a player with 1 vote, but about 12 times as much. In the game $[17 ; 9,8,5,4,4,1,1]$, a player with 9 votes does not get 9 times as much as a player with 1 vote, but about 20 times as much. Figure 3 illustrates how relative payoffs deviate very substantially from relative weights.

These examples and the ones in the online appendix show a similar pattern. On the one hand, many of the larger players have an expected payoff close to $w_{i}$, such that their predicted payoff as coalition partners is similar to their weights. On the other hand, the implied exchange rates between players may deviate substantially from the relative weights, and this is often the case when the smallest player type is involved.

\section{Qualitative equilibrium phenomena}

An alternative way of looking at the importance of deviations is to focus not on their size, but on the presence of equilibrium phenomena that would be ruled out if payoffs were proportional to the MIWs. I discuss three such phenomena: players that are not interchangeable may have the same expected equilibrium payoffs, some players may be too expensive to receive any proposals, and 
surplus coalitions may form. All three phenomena can be illustrated using one of the weighted majority games in the dataset, $[13 ; 7,6,4,3,3,1]$, corresponding to Belgium in 1972.

Example 7 Consider the weighted majority game $[13 ; 7,6,4,3,3,1]$. Note that types [7] and [6] are genuinely asymmetric: coalition [733] is winning but coalition [633] is losing. Likewise, types [4] and [3] are genuinely asymmetric because [643] is winning but [633] is losing. Let $\theta=\left(\frac{7}{24}, \frac{6}{24}, \frac{4}{24}, \frac{3}{24}, \frac{3}{24}, \frac{1}{24}\right)$. All $S S P E$ have $v_{[7]}=v_{[6]}=\frac{46}{164}, v_{[4]}=v_{[3]}=\frac{23}{164}$ and $v_{[1]}=\frac{3}{164}$.

Proof. See Online Appendix.

\section{Asymmetric players may have the same payoff}

Example 7 shows that it is possible for two players to have the same equilibrium expected payoffs, even though one of the players is more valuable as a coalition partner and has the additional advantage of a higher recognition probability.

Some intuition for this result can be obtained by inspecting the list of MWCs. There are seven MWCs of five types: [76], [743], [733], [643], [6331]. All MWCs have exactly 13 votes except for the two coalitions of type [743]. If $v_{[7]}>v_{[6]}$ and $v_{[4]}>v_{[3]}$, coalition [743] would be too expensive to be proposed by any player type, because the alternative coalitions [643] and [733] would be cheaper. This leaves four coalition types that could potentially be proposed, [76], [733], [643] and [6331]. Given this list, player [1] would need the cooperation of player [6] and both players of type [3], whereas player [4] would need player [6] and one of the players of type [3]. Likewise, player [7] would need either player [6] or both players of type [3]. Types [6] and [3] would be more in demand than types [7] and [4], and it would not be possible 
to have $v_{[7]}>v_{[6]}$ and $v_{[4]}>v_{[3]}$. It turns out that, in equilibrium, $v_{[7]}=v_{[6]}$ and $v_{[4]}=v_{[3]}$.

\section{Some players may be too expensive to receive proposals}

The equalities $v_{[7]}=v_{[6]}$ and $v_{[4]}=v_{[3]}$ have two implications. On the one hand, coalition type [743] becomes relevant, because it is just as expensive as [643] and [733] despite having one more vote. On the other hand, coalition type [6331] becomes too expensive for anybody other than player [1] even though it only has 13 votes. Players [6] and [3] would rather propose [643] than [6331], since its total cost is $v_{[6]}+v_{[4]}+v_{[3]}=v_{[6]}+2 v_{[3]}<v_{[6]}+2 v_{[3]}+v_{[1]}$.

Note that the substitutability logic applies to this example, but in a somewhat perverse way. Instead of applying to the MWCs with 13 votes (coalition types [76], [733], [643], and [6331]), it applies to coalition types [76], [733], [643] and [743].

Looking at the SSPE payoffs, player [1] appears underpriced since it only expects about 0.02 even though its weight share is about 0.04. Types [6] and [3] are getting a disproportionately high payoff compared to their weight share but this does not result in their exclusion; instead, it is type [1] that is excluded. Indeed, given that $v_{[7]}=v_{[6]}$ and $v_{[4]}=v_{[3]}$, player type [1] would be perceived as too expensive for any positive value of $v_{[1]}$.

\section{Surplus coalitions may form}

The original Baron-Ferejohn model with symmetric players always leads to minimal winning coalitions, since the proposer could otherwise drop one of the responders and still have a winning coalition. With asymmetric players, it is still true that all coalition partners must be pivotal, but the proposer is not necessarily pivotal. In the previous example, type [1] finds it optimal to 
propose surplus coalitions such as [7331] or [7431], since they are as expensive as the minimal winning coalition [6331] given that $v_{[7]}=v_{[6]}$ and $v_{[4]}=v_{[3]}$. Hence, surplus coalitions are not ruled out in equilibrium under weighted voting, though the only type of surplus coalition that may form is one in which the proposer is the only member of the coalition who is not pivotal.

\section{Concluding remarks}

This paper provides a necessary and sufficient condition for equilibrium payoffs to be proportional in the Baron-Ferejohn model with weighted voting. When the condition is satisfied, the set of available coalitions is sufficiently rich so that none of the parties appears systematically too often (excess demand) or too seldom (excess supply) in the final coalition. The condition is relatively easy to check in applications since all equations involved are linear. Using the condition, it is found that the frequency of legislatures in the field with proportional equilibrium payoffs is about $69 \%$, though there is a lot of variation across countries. This frequency may be viewed as sufficiently high to support empirical work, specially in the countries where it is highest.

Most counterexamples are not a result of the competitive bargaining logic failing, but rather of its working in unexpected ways. However, in these cases the deviations from proportionality may be substantial, both quantitatively and qualitatively, as the examples provided illustrate.

\section{References}

[1] Ansolabehere, Stephen, James M. Snyder, Aaron B. Strauss, and Michael M. Ting. 2005. "Voting Weights and Formateur Advantages in the For- 
mation of Coalition Governments." American Journal of Political Science 49: 550-563.

[2] Banks, Jeffrey S., and John Duggan. 2000. "A Bargaining Model of Collective Choice." American Political Science Review 94: 73-88.

[3] Baron, David P., and John A. Ferejohn. 1989. "Bargaining in Legislatures." American Political Science Review 83: 1181-1206.

[4] Baron, David P., and Ehud Kalai. 1993. "The Simplest Equilibrium of a Majority-Rule Division Game." Journal of Economic Theory 61: 290-301.

[5] Bondareva, Olga. 1963. Some Applications of Linear Programming to the Theory of Cooperative Games. Problemy Kibernetiki 10: 119-39 [in Russian].

[6] Cutler, Josh, Scott De Marchi, Max Gallop, Florian M. Hollenbach, Michael Laver, and Matthias Orlowski. 2014. "Cabinet Formation and Portfolio Distribution in European Multiparty Systems." British Journal of Political Science 46: 31-43.

[7] Derks, Jean, and Jeroen Kuipers. 1997. "Implementing the Simplex Method for Computing the Prenucleolus of Transferable Utility Games." Maastricht University. Unpublished manuscript.

[8] Diermeier, Daniel, and Antonio Merlo. 2004. "An Empirical Investigation of Coalitional Bargaining Procedures." Journal of Public Economics 88: 783-797.

[9] Eraslan, Hülya, and Andrew McLennan. 2013. "Uniqueness of Stationary Equilibrium Payoffs in Coalitional Bargaining." Journal of Economic Theory 148: 2195-2222. 
[10] Freixas, Josep, and Xavier Molinero. 2009. "On the Existence of a Minimum Integer Representation for Weighted Voting Systems." Annals of Operations Research 166: 243-260.

[11] Jeon, Jee S. 2015. "The Emergence and Persistence of Oligarchy: A Dynamic Model of Endogenous Political Power." Florida State University. Unpublished manuscript.

[12] Kalandrakis, Tasos. 2004. "A Three-Player Dynamic Majoritarian Bargaining Game." Journal of Economic Theory 116: 294-322.

[13] Kalandrakis, Tasos. 2006. "Proposal Rights and Political Power." American Journal of Political Science, 50: 441-448.

[14] Kopelowitz, Alexander. 1967. "Computation of the Kernels of Simple Games and of the Nucleolus of n-Person Games." RM-31, Hebrew University of Jerusalem.

[15] Kurz, Sascha, Stefan Napel, and Andreas Nohn. 2014. "The Nucleolus of Large Majority Games." Economics Letters 123: 139-143.

[16] Laver, Michael, Scott De Marchi, and Hande Mutlu .2011. "Negotiation in Legislatures over Government Formation." Public Choice 147: 285-304.

[17] Le Breton, Michel, Maria Montero, and Vera Zaporozhets. 2012. "Voting Power in the EU Council of Ministers and Fair Decision Making in Distributive Politics." Mathematical Social Sciences 63: 159-173.

[18] Montero, Maria. 2000. Endogenous Coalition Formation and Bargaining. Open access publications from Tilburg University. 
[19] Montero, Maria. 2006. "Noncooperative Foundations of the Nucleolus in Majority Games." Games and Economic Behavior 54: 380-97.

[20] Napel, Stefan, and Mika Widgrén. 2001. "Inferior Players in Simple Games." International Journal of Game Theory 30: 209-220.

[21] Okada, Akira. 1996. "A Noncooperative Coalitional Bargaining Game with Random Proposers." Games and Economic Behavior 16: 97-108.

[22] Ostmann, Axel. 1987. "On the Minimal Representation of Homogeneous Games." International Journal of Game Theory, 16, 69-81.

[23] Peleg, Bezalel. 1968. "On Weights of Constant-Sum Majority Games." SIAM Journal of Applied Mathematics 16: 527-532.

[24] Peleg, Bezalel, and Joachim Rosenmüller. 1992. "The Least Core, Nucleolus and Kernel of Homogeneous Weighted Majority Games." Games and Economic Behavior 4: 588-605.

[25] Schmeidler, David. 1969. "The Nucleolus of a Characteristic Function Game." SIAM Journal on Applied Mathematics 17: 1163-1170.

[26] Shapley, Lloyd S. 1967. "On Balanced Sets and Cores." Naval Research Logistics Quarterly 9: 45-8.

[27] Snyder, James M., Michael M. Ting, and Stephen Ansolabehere. 2005. "Legislative Bargaining under Weighted Voting." American Economic Review, 95: 981-1004.

[28] Vanderbei, Robert J. 2008. Linear Programming: Foundations and Extensions. International Series in Operations Research and Management Science, Springer. 
[29] Warwick, Paul V., and James N. Druckman. 2006. "The Portfolio Allocation Paradox: An Investigation into the Nature of a very Strong but Puzzling Relationship." European Journal of Political Research 45: 635665.

[30] Winter, Eyal. 1996. "Voting and Vetoing." American Political Science Review 90: 813-823.

[31] Yan, Huibin. 2002. "Noncooperative Selection of the Core." International Journal of Game Theory 31: 527-540. 


\title{
Online Appendix to "Proportional payoffs in legislative bargaining with weighted voting: a characterization"
}

\author{
Maria Montero
}

\section{Calculations for $[5 ; 3,2,2,1]$}

By contradiction, suppose $v=\left(\frac{3}{8}, \frac{2}{8}, \frac{2}{8}, \frac{1}{8}\right)$. What would be the optimal proposer behavior given $v$ ? The player with 3 votes needs to buy 2 votes, hence it always offers $v_{[2]}$ to one of the players with 2 votes (the player with 1 vote is of no use to this player, regardless of the value of $\left.v_{[1]}\right)$. The player with 1 vote needs to buy 4 votes, and buys them from the two players that control 2 votes each. A player with 2 votes needs to buy 3 votes, and is indifferent between buying them from the large player or from the other two players since $v_{[3]}=v_{[2]}+v_{[1]}$. Let $p$ be the probability that a player with 2 votes proposes to the player with 3 votes (conditional on a player with 2 votes being selected as proposer). Expected payoffs for types [3] and [1] must satisfy the following equations:

$$
\begin{aligned}
& \frac{3}{8}=\frac{3}{8}\left[1-\frac{2}{8}\right]+\frac{4}{8} p \frac{3}{8} \\
& \frac{1}{8}=\frac{1}{8}\left[1-\frac{4}{8}\right]+\frac{4}{8}(1-p) \frac{1}{8}
\end{aligned}
$$


From the second equation we find $p=0$. This means that in order to sustain a payoff of $\frac{1}{8}$ for type [1], type [2] must always propose a coalition of type [221]. However, $p=0$ does not solve the first equation: in order to sustain a payoff of $\frac{3}{8}$ for type [3], $p$ must be $\frac{1}{2}$.

Interestingly, the equilibrium is still competitive in the sense that $v_{[3]}=$ $v_{[2]}+v_{[1]}$. Below we construct an equilibrium strategy profile. Let player [3] propose to each of the two players of type [2] with probability $\frac{1}{2}$, and let each of the players of type [2] propose to player [3] with probability $p$. The equilibrium values of $v_{[3]}, v_{[2]}, v_{[1]}$ and $p$ can be found from the following system:

$$
\begin{aligned}
v_{[3]} & =\frac{3}{8}\left[1-v_{[2]}\right]+\frac{4}{8} p v_{[3]} \\
v_{[2]} & =\frac{2}{8}\left[p\left(1-v_{[3]}\right)+(1-p)\left(1-v_{[2]}-v_{[1]}\right)\right]+\frac{3}{8} \frac{1}{2} v_{[2]}+\frac{2}{8}(1-p) v_{[2]}+\frac{1}{8} v_{[2]} \\
v_{[1]} & =\frac{1}{8}\left[1-2 v_{[2]}\right]+\frac{4}{8}(1-p) v_{[1]} \\
v_{[3]} & =v_{[2]}+v_{[1]}
\end{aligned}
$$

The solution to this system is $v_{[3]}=\frac{5}{14}, v_{[2]}=\frac{4}{14}, v_{[1]}=\frac{1}{14}$ and $p=\frac{1}{2}$. This is an equilibrium since players are behaving optimally both as proposers and as responders. Because of the uniqueness result of Eraslan and McLennan (2013), all SSPE must have the same payoff vector.

\section{Calculations for $[13 ; 7,6,4,3,3,1]$}

There are seven MWCs of five types: [76], [743], [733], [643], [6331]. If expected equilibrium payoffs were proportional, only types [76], [733], [643] and [6331] could be proposed in equilibrium. It can be checked that the necessary and sufficient condition for proportionality does not hold: for any probability distribution over those coalitions, type [6] and/or type [3] would appear in the final coalition disproportionately often. 
It turns out that, even though there are five player types, SSPE payoffs divide the players in only three groups, which we denote as $L, M$ and $S$. We now construct an equilibrium with $v_{[7]}=v_{[6]}:=v_{L}, v_{[4]}=v_{[3]}:=v_{M}, v_{[1]}:=v_{S}$ and $v_{L}=2 v_{M}$. In this situation, player [7] is indifferent between proposing to the other large player and paying $v_{L}$, and proposing to two medium players, paying $v_{M}$ to each $\left(v_{L}\right.$ in total). Type [6] is also indifferent between buying votes from the large player or from two medium players (except that, when buying votes from a medium player, one of the two medium players has to be of type [4] because otherwise the coalition would be losing). Coalition [6331] would be too expensive, since on top of $2 v_{M}$ one needs to pay $v_{S}>0$. Type [4] may propose [743] or [643]; in both cases it needs to pay $v_{L}+v_{M}$. Likewise, type [3] has three coalition types that are equally optimal: [743], [733] and [643]; coalition [6331] is too expensive. Player [1] has [6331] as its only MWC; given the prices, it could replace [6] with [7] or/and [3] with [4] at no extra cost, hence the surplus coalitions [7331] and [7431] would also be optimal for type [1] (we return to this point below).

We now construct a profile of SSPE strategies. The following table introduces a notation for the strategies. The rows in the table are player types and the columns are coalition types. Each entry in the table represents the probability that the player type in the corresponding row proposes the coalition type in the corresponding column. It is assumed that all players of the same type follow the same strategy and each coalition of the same type is proposed 
with equal probability.

$$
\begin{array}{cccccc} 
& {[76]} & {[743]} & {[733]} & {[643]} & {[6331]} \\
{[7]} & \alpha & \beta & 1-\alpha-\beta & - & - \\
{[6]} & \gamma & - & - & 1-\gamma & 0 \\
{[4]} & - & \mu & - & 1-\mu & - \\
{[3]} & - & \pi & \rho & 1-\pi-\rho & 0 \\
{[1]} & - & - & - & - & 1
\end{array}
$$

Equilibrium strategies and payoffs solve the following system of equations ${ }^{1}$

$$
\begin{aligned}
v_{L} & =\frac{7}{24}\left[1-v_{L}\right]+\left[\frac{6}{24} \gamma+\frac{4}{24} \mu+\frac{6}{24}(\pi+\rho)\right] v_{L} \\
v_{L} & =\frac{6}{24}\left[1-v_{L}\right]+\left[\frac{7}{24} \alpha+\frac{4}{24}(1-\mu)+\frac{6}{24}(1-\pi-\rho)+\frac{1}{24}\right] v_{L} \\
v_{M} & =\frac{4}{24}\left[1-v_{L}-v_{M}\right]+\left[\frac{7}{24} \beta+\frac{6}{24}(1-\gamma)+\frac{6}{24}(1-\rho)\right] v_{M} \\
v_{M} & =\frac{3}{24}\left[1-v_{L}-v_{M}\right]+\left[\frac{7}{24}\left(\frac{\beta}{2}+1-\alpha-\beta\right)+\frac{6}{24} \frac{1-\gamma}{2}+\frac{4}{24} \frac{1}{2}+\frac{3}{24} \rho+\frac{1}{24}\right] v_{M} \\
v_{S} & =\frac{1}{24}\left[1-v_{L}-2 v_{M}\right] \\
v_{L} & =2 v_{M}
\end{aligned}
$$

There are many solutions to this system, all with $v_{L}=\frac{46}{164}, v_{M}=\frac{23}{164}$ and $v_{S}=\frac{3}{164}$. The mixed strategies are not uniquely determined. A possible solution is $\alpha=\mu=\pi=0, \beta=\frac{5}{23}, \gamma=\frac{14}{23}, \rho=\frac{55}{138}$. These strategies constitute an SSPE since players are behaving optimally both as proposers and as responders: only optimal coalitions are proposed given the acceptance thresholds

\footnotetext{
${ }^{1}$ Note that we are simplifying the first five equations by using the sixth one (i.e., all coalitions proposed with positive probability in equilibrium must give the same payoff to the proposer). For example, player [6]'s proposer payoff is written as $1-v_{L}$ rather than $\gamma\left[1-v_{L}\right]+(1-\gamma)\left[1-2 v_{M}\right]$.
} 
$\left(v_{L}, v_{M}\right.$ and $\left.v_{S}\right)$, and the acceptance thresholds equal the continuation values given the strategies. Due to the uniqueness result of Eraslan and McLennan (2013), all SSPE must have the same $v$-values.

There are also equilibria in which surplus coalitions are proposed with positive probability. For example, if type [1] proposes [6331] with probability $\frac{1}{2}$ and [7431] with probability $\frac{1}{2}$, the system of equations can be amended accordingly and a new solution for the equilibrium strategies is $\alpha=\mu=\pi=0$, $\beta=\frac{12}{161}, \gamma=\frac{14}{23}, \rho=\frac{29}{92}$ (the $v$-values are of course unaffected).

\section{Predicted size of the deviations}

The following tables compare equilibrium payoffs and weights for all games in the dataset with at most 7 players that fail to satisfy the condition (excluding games with a veto player, of which there are two in the database). For each game, the tables shows $w_{i}$ (the MIWs), $v_{i}$ (expected equilibrium payoffs), and two quantitative measures of how far $v$ is from being proportional to $w$. One such measure is $\frac{v_{i}}{w_{i} / \sum_{j \in N} w_{j}}$, the ratio of payoffs to weights, where weights are normalized so that they add up to 1 . This ratio measures how much of a player's weight is translated into expected equilibrium payoffs; if expected equilibrium payoffs were proportional to weights it would always be 1 . Another measure is the relative payoffs $v_{i} / v_{n}$, i.e. the exchange rate between players according to equilibrium predictions. If expected equilibrium payoffs were proportional, this exchange rate would always be equal to $w_{i} / w_{n}$ (in particular, if $w_{n}=1$, this ratio would replicate the MIWs).

Expected payoffs for individual players can be substantially different from weight shares, and this is very often true for the smallest player type, who may get as little as $43 \%$ of its weight share. As a result, ratios between a player's 
payoff and the payoff of the smallest player are often very different from $w_{i} / w_{n}$. Nevertheless, if we focus on the ratio of expected payoffs to weights, we see that many players have a ratio close to 1 .

Table A1. Homogeneous games with up to 6 players

$\begin{array}{ccccccc}\text { Weights } & \mathbf{7} & \mathbf{5} & \mathbf{5} & \mathbf{2} & \mathbf{2} & \mathbf{1} \\ \text { Payoffs } & 0.323 & 0.226 & 0.226 & 0.097 & 0.097 & 0.032 \\ \text { Payoffs/weights } & 1.014 & 0.993 & 0.993 & 1.067 & 1.067 & 0.699 \\ \text { Relative payoffs } & 10.16 & 7.10 & 7.10 & 3.05 & 3.05 & 1 \\ & & & & & & \\ \text { Weights } & \mathbf{5} & \mathbf{4} & \mathbf{4} & \mathbf{1} & \mathbf{1} & \mathbf{1} \\ \text { Payoffs } & 0.324 & 0.297 & 0.297 & 0.027 & 0.027 & 0.027 \\ \text { Payoffs/weights } & 1.038 & 1.190 & 1.190 & 0.430 & 0.430 & 0.430 \\ \text { Relative payoffs } & 12.06 & 11.06 & 11.06 & 1 & 1 & 1 \\ & & & & & & \\ \text { Weights } & \mathbf{5} & \mathbf{3} & \mathbf{3} & \mathbf{2} & \mathbf{1} & \\ \text { Payoffs } & 0.376 & 0.208 & 0.208 & 0.168 & 0.040 & \\ \text { Payoffs/weights } & 1.053 & 0.970 & 0.970 & 1.178 & 0.556 & \\ \text { Relative payoffs } & 9.47 & 5.24 & 5.24 & 4.24 & 1 & \\ & & & & & & \\ \text { Weights } & \mathbf{5} & \mathbf{2} & \mathbf{2} & \mathbf{2} & \mathbf{1} & \\ \text { Payoffs } & 0.412 & 0.176 & 0.176 & 0.176 & 0.059 & \\ \text { Payoffs/weights } & 0.988 & 1.059 & 1.059 & 1.059 & 0.706 & \\ \text { Relative payoffs } & 7 & 3 & 3 & 3 & 1 & \\ & & & & & & \\ \text { Weights } & \mathbf{4} & \mathbf{3} & \mathbf{3} & \mathbf{1} & \mathbf{1} & \\ \text { Payoffs } & 0.333 & 0.295 & 0.295 & 0.038 & 0.038 & \\ \text { Payoffs/weights } & 1.000 & 1.181 & 1.181 & 0.456 & 0.456 & \\ \text { Relative payoffs } & 8.77 & 7.77 & 7.77 & 1 & 1 & \\ \text { Weights } & \mathbf{3} & \mathbf{2} & \mathbf{2} & \mathbf{1} & & \\ \text { Payoffs } & 0.357 & 0.286 & 0.286 & 0.071 & & \\ \text { Payoffs/weights } & 0.952 & 1.143 & 1.143 & 0.571 & & \\ \text { Relative payoffs } & 5 & 4 & 4 & 1 & & \\ & & & & & & \\ \text { Pats } & & & & \end{array}$


Table A2. Homogeneous games with 7 players

$\begin{array}{cccccccc}\text { Weights } & \mathbf{9} & \mathbf{7} & \mathbf{7} & \mathbf{2} & \mathbf{2} & \mathbf{2} & \mathbf{1} \\ \text { Payoffs } & 0.302 & 0.233 & 0.233 & 0.069 & 0.069 & 0.069 & 0.026 \\ \text { Payoffs/weights } & 1.006 & 0.998 & 0.998 & 1.035 & 1.035 & 1.035 & 0.771 \\ \text { Relative payoffs } & 11.74 & 9.06 & 9.06 & 2.69 & 2.69 & 2.69 & 1 \\ \text { Weights } & \mathbf{9} & \mathbf{6} & \mathbf{6} & \mathbf{3} & \mathbf{2} & \mathbf{1} & \mathbf{1} \\ \text { Payoffs } & 0.325 & 0.217 & 0.217 & 0.108 & 0.085 & 0.024 & 0.024 \\ \text { Payoffs/weights } & 1.013 & 1.013 & 1.013 & 1.013 & 1.188 & 0.661 & 0.661 \\ \text { Relative payoffs } & 13.79 & 9.19 & 9.19 & 4.60 & 3.60 & 1 & 1 \\ \text { Weights } & \mathbf{9} & \mathbf{3} & \mathbf{3} & \mathbf{3} & \mathbf{2} & \mathbf{1} & \mathbf{1} \\ \text { Payoffs } & 0.416 & 0.139 & 0.139 & 0.139 & 0.109 & 0.023 & 0.023 \\ \text { Payoffs/weights } & 1.017 & 1.017 & 1.017 & 1.017 & 1.200 & 0.650 & 0.650 \\ \text { Relative payoffs } & 14.08 & 4.69 & 4.69 & 4.69 & 3.69 & 1 & 1\end{array}$

Table A3. Nonhomogeneous games with up to 6 players

$\begin{array}{ccccccc}\text { Weights } & \mathbf{9} & \mathbf{5} & \mathbf{5} & \mathbf{3} & \mathbf{2} & \mathbf{2} \\ \text { Payoffs } & 0.364 & 0.182 & 0.182 & 0.091 & 0.091 & 0.091 \\ \text { Payoffs/weights } & 1.051 & 0.945 & 0.945 & 0.788 & 1.182 & 1.182 \\ \text { Relative payoffs } & 4 & 2 & 2 & 1 & 1 & 1 \\ \text { Weights } & \mathbf{8} & \mathbf{6} & \mathbf{5} & \mathbf{3} & \mathbf{3} & \mathbf{1} \\ \text { Payoffs } & 0.320 & 0.227 & 0.206 & 0.113 & 0.113 & 0.020 \\ \text { Payoffs/weights } & 1.039 & 0.983 & 1.073 & 0.983 & 0.983 & 0.528 \\ \text { Relative payoffs } & 15.75 & 11.16 & 10.16 & 5.58 & 5.58 & 1 \\ \text { Weights } & \mathbf{7} & \mathbf{6} & \mathbf{4} & \mathbf{3} & \mathbf{3} & \mathbf{1} \\ \text { Payoffs } & 0.280 & 0.280 & 0.140 & 0.140 & 0.140 & 0.018 \\ \text { Payoffs/weights } & 0.962 & 1.122 & 0.841 & 1.122 & 1.122 & 0.439 \\ \text { Relative payoffs } & 15.33 & 15.33 & 7.67 & 7.67 & 7.67 & 1 \\ \text { Weights } & \mathbf{5} & \mathbf{4} & \mathbf{3} & \mathbf{2} & \mathbf{2} & \\ \text { Payoffs } & 0.290 & 0.280 & 0.150 & 0.140 & 0.140 & \\ \text { Payoffs/weights } & 0.928 & 1.119 & 0.801 & 1.119 & 1.119 & \\ \text { Relative payoffs } & 2.07 & 2 & 1.07 & 1 & 1 & \end{array}$


Table A4. Nonhomogeneous games with 7 players

$\begin{array}{cccccccc}\text { Weights } & \mathbf{1 3} & \mathbf{1 1} & \mathbf{9} & \mathbf{6} & \mathbf{5} & \mathbf{4} & \mathbf{2} \\ \text { Payoffs } & 0.261 & 0.218 & 0.174 & 0.130 & 0.088 & 0.087 & 0.043 \\ \text { Payoffs/weights } & 1.003 & 0.989 & 0.968 & 1.082 & 0.877 & 1.082 & 1.082 \\ \text { Relative payoffs } & 6.03 & 5.03 & 4.03 & 3 & 2.03 & 2 & 1 \\ \text { Weights } & \mathbf{1 3} & \mathbf{1 0} & \mathbf{9} & \mathbf{6} & \mathbf{6} & \mathbf{3} & \mathbf{1} \\ \text { Payoffs } & 0.264 & 0.198 & 0.198 & 0.132 & 0.132 & 0.066 & 0.010 \\ \text { Payoffs/weights } & 0.975 & 0.951 & 1.056 & 1.056 & 1.056 & 1.056 & 0.472 \\ \text { Relative payoffs } & 26.86 & 20.14 & 20.14 & 13.43 & 13.43 & 6.71 & 1 \\ \text { Weights } & \mathbf{1 2} & \mathbf{1 0} & \mathbf{7} & \mathbf{5} & \mathbf{4} & \mathbf{3} & \mathbf{1} \\ \text { Payoffs } & 0.288 & 0.237 & 0.170 & 0.119 & 0.102 & 0.068 & 0.017 \\ \text { Payoffs/weights } & 1.009 & 0.996 & 1.017 & 0.997 & 1.070 & 0.947 & 0.704 \\ \text { Relative payoffs } & 17.20 & 14.16 & 10.12 & 7.08 & 6.08 & 4.04 & 1 \\ \text { Weights } & \mathbf{1 1} & \mathbf{8} & \mathbf{7} & \mathbf{4} & \mathbf{4} & \mathbf{1} & \mathbf{1} \\ \text { Payoffs } & 0.320 & 0.222 & 0.209 & 0.111 & 0.111 & 0.014 & 0.014 \\ \text { Payoffs/weights } & 1.046 & 1.000 & 1.073 & 1.000 & 1.000 & 0.491 & 0.491 \\ \text { Relative payoffs } & 23.43 & 16.28 & 15.28 & 8.14 & 8.14 & 1 & 1 \\ \text { Weights } & \mathbf{1 0} & \mathbf{9} & \mathbf{7} & \mathbf{3} & \mathbf{3} & \mathbf{3} & \mathbf{1} \\ \text { Payoffs } & 0.269 & 0.269 & 0.179 & 0.090 & 0.090 & 0.090 & 0.013 \\ \text { Payoffs/weights } & 0.969 & 1.077 & 0.923 & 1.077 & 1.077 & 1.077 & 0.462 \\ \text { Relative payoffs } & 21 & 21 & 14 & 7 & 7 & 7 & 1 \\ \text { Weights } & \mathbf{1 0} & \mathbf{3} & \mathbf{3} & \mathbf{3} & \mathbf{2} & \mathbf{2} & \mathbf{1} \\ \text { Payoffs } & 0.426 & 0.120 & 0.120 & 0.120 & 0.093 & 0.093 & 0.027 \\ \text { Payoffs/weights } & 1.023 & 0.961 & 0.961 & 0.961 & 1.117 & 1.117 & 0.649 \\ \text { Relative payoffs } & 15.76 & 4.44 & 4.44 & 4.44 & 3.44 & 3.44 & 1 \\ \text { Weights } & \mathbf{9} & \mathbf{8} & \mathbf{5} & \mathbf{4} & \mathbf{4} & \mathbf{1} & \mathbf{1} \\ \text { Payoffs } & 0.278 & 0.278 & 0.139 & 0.139 & 0.139 & 0.014 & 0.014 \\ \text { Payoffs/weights } & 0.988 & 1.111 & 0.889 & 1.111 & 1.111 & 0.444 & 0.444 \\ \text { Relative payoffs } & 20 & 20 & 10 & 10 & 10 & 1 & 1\end{array}$




\section{An alternative way of checking the condition using linear programming}

Consider the following linear programming problem

$$
\begin{aligned}
& \min e \\
\text { s.t. } \sum_{i \in S} x_{i}+e & \geq 1 \text { for all } S \in W \\
\sum_{i \in N} x_{i} & =1 \\
x_{i} & \geq 0 \text { for all } i \in N ; e \geq 0
\end{aligned}
$$

Its interpretation is the following. Take any $\left(x_{1}, \ldots, x_{n}\right)$ vector, and any winning coalition $S$. Coalition $S$ can divide the dollar by itself, but it is getting only $\sum_{i \in S} x_{i}$ in this particular allocation. The difference $1-\sum_{i \in S} x_{i}$ is known as the excess of the coalition, though perhaps deficit would be a better term. The linear program above finds allocations $x$ that minimize the maximum excess. $^{2}$ This linear programming problem is well known in cooperative game theory and is related to the core (in particular, if the solution has $e=0$, the core is nonempty; this is not the case in weighted majority games unless there are veto players).

The following result is adapted from Peleg and Rosenmüller's (1992) theorems 3.2 and 3.3, which concern the set $W^{m}$ and homogeneous games.

Claim 1 Let $\left[q ; w_{1}, \ldots, w_{n}\right]$ be an arbitrary weighted majority game, normalized so that $\sum_{i \in N} w_{i}=1$. Then $W^{*}$ is weakly balanced if and only if $x=w$ and $e=1-\bar{q}$ solve linear programming problem (1).

\footnotetext{
${ }^{2}$ Rewriting $\sum_{i \in S} x_{i}+e \geq 1$ as $e \geq 1-\sum_{i \in S} x_{i}$, we see that the inequalities impose that excesses of the winning coalitions are at most $e$. This number $e$ is then minimized.
} 
This result allows us to check the weak balancedness of $W^{*}$ by solving (1) and comparing the optimal value of $e$ with $1-\bar{q}$.

To see that claim 1 is correct, construct the dual program of (1) (see, for example, Vanderbei (2008), chapter 5), where $\lambda_{S}$ is the dual variable associated to the constraint $\sum_{i \in S} x_{i}+e \geq 1$ and $\mu$ is the dual variable associated to $\sum_{i \in N} x_{i}=1$ (rewritten as $\sum_{i \in N} x_{i} \leq 1$, or equivalently as $-\sum_{i \in N} x_{i} \geq-1$ ).

$$
\max \sum_{S \in W} \lambda_{S}-\mu
$$

$$
\begin{aligned}
\text { s. t. } \sum_{S \in W, S \ni i} \lambda_{S}-\mu & \leq 0 \text { for all } i \in N \\
\sum_{S \in W} \lambda_{S} & \leq 1 \\
\lambda_{S} & \geq 0 \text { for } S \in W, \mu \geq 0 .
\end{aligned}
$$

The complementary slackness theorem (see theorem 5.3 in Vanderbei (2008)) tells us that a pair of feasible solutions for the primal (1) and for the dual (2) are optimal for their respective problems if and only if $\lambda_{S}\left(1-\sum_{i \in S} x_{i}-e\right)=0$ for all $S \in W, \mu\left(1-\sum_{i \in N} x_{i}\right)=0, x_{i}\left(\sum_{S \in W, S \ni i} \lambda_{S}-\mu\right)=0$ for all $i \in N$, and $e\left(1-\sum_{S \in W} \lambda_{S}\right)=0$.

We now prove claim 1.

1. Sufficiency. Suppose $W^{*}$ is weakly balanced. Then we can construct feasible solutions for the primal and for the dual such that the complementary slackness conditions are satisfied. For the primal, let $x=w$ and $e=1-\bar{q}$. This is clearly feasible for the primal since by definition $\bar{q}=\min _{S \in W} w_{i}$, hence $\sum_{i \in S} w_{i}+(1-\bar{q}) \geq 1$ for all $S \in W$. As for the dual, we can construct $\lambda_{S}$ in the same way we constructed $p(S)$ in the proof of the main proposition. Since $W^{*}$ is weakly balanced, there are balancing weights $\left(\lambda_{S}^{\prime}\right)_{S \in W^{*}}$ such that $\sum_{S \in W^{*}, S \ni i} \lambda_{S}^{\prime}=1$ for all $i \in N$. Now construct $\lambda_{S}$ in the following way. Draw a player at random from $i$ using $w$ as probability vector, and, 
given $i$, draw a coalition $S \in W^{*}, S \ni i$ at random using $\left(\lambda_{S}^{\prime}\right)_{S \in W^{*}, S \ni i}$. For any $S \in W$, denote by $\lambda_{S}$ the probability that $S$ is drawn given this procedure. Clearly, $\sum_{S \in W} \lambda_{S}=1$ (since the process always draws exactly one coalition), $\lambda_{S}>0$ implies $S \in W^{*}$ (since only coalitions in $W^{*}$ have been considered), and $\sum_{S \ni i} \lambda_{S}=\bar{q}$ (the probability that $i$ appears in the final coalition is $\left.\sum_{S \ni i} \sum_{j \in S} w_{j} \lambda_{S}^{\prime}=\sum_{S \in W^{*}, S \ni i} \sum_{j \in S} w_{j} \lambda_{S}^{\prime}=\sum_{S \in W^{*}, S \ni i} \bar{q} \lambda_{S}^{\prime}=\bar{q}\right)$. Take the $\left(\lambda_{S}\right)_{S \in W}$ constructed in this way and $\mu=\bar{q}$ as feasible solutions for the dual. They are clearly feasible, and moreover $\sum_{S \in W, S \ni i} \lambda_{S}-\mu \leq 0$ for all $i \in N$ and $\sum_{S \in W} \lambda_{S} \leq 1$ both hold with equality, which immediately implies two of the complementary slackness conditions, $x_{i}\left(\sum_{S \in W, S \ni i} \lambda_{S}-\mu\right)=0$ for all $i \in N$, and $e\left(1-\sum_{S \in W} \lambda_{S}\right)=0$. The other two conditions are also immediate: by construction, $\lambda_{S}>0$ implies $\sum_{i \in S} w_{i}=\bar{q}$. We have also assumed that weights are normalized, hence $1=\sum_{i \in N} w_{i}$.

2. Necessity. Suppose $x=w$ and $e=1-\bar{q}$ solve the primal program, in which case the optimal value of the primal is $1-\bar{q}$. By the strong duality theorem (see Vanderbei, 2008, theorem 5.2) the dual program also has a solution (and the optimal value of the objective function in the dual problem is also $1-\bar{q})$. Since both the primal and the dual have a solution, the complementary slackness conditions must be satisfied for $x=w, e=1-\bar{q}$ and some suitable values of $\lambda_{S}$ and $\mu$. According to the complementary slackness conditions, if $\lambda_{S}>0$, then $1-\sum_{i \in S} w_{i}=1-\bar{q}$, that is, only coalitions in $W^{*}$ have a positive value of $\lambda_{S}$. Also, $w_{i}>0$ implies $\sum_{S \in W, S \ni i} \lambda_{S}=\mu$ for $i$, which, since only coalitions in $W^{*}$ have a positive weight, can be written as $\sum_{S \in W^{*}, S \ni i} \lambda_{S}=\mu$.

If the weighted majority game is such that $\bar{q}=1$, we are in the trivial case in which a winning coalition requires the presence of all players with positive weight. Then the optimal value of the primal is 0 , and the optimal value of the dual is 0 . This is a trivial case in which the set $W^{*}$ is clearly weakly balanced 
since one can place a weight of 1 on the grand coalition and 0 on all others.

Let $\bar{q}<1$. Then the optimal value of the primal is positive, and the optimal value of the dual must be positive as well. This in turn requires that $\mu>0$ (if $\mu=0$, feasibility of the dual program would imply $\lambda_{S}=0$ for all $S \in W$, and the value of the objective function of the dual program would be 0). We can then construct weights $\lambda_{S}^{\prime}=\frac{\lambda_{S}}{\mu}$. Are these weights balancing weights? If $w_{i}>0$, complementary slackness requires that $\sum_{S \in W, S \ni i} \lambda_{S}=\mu$, or equivalently that $\sum_{S \in W^{*}, S \ni i} \lambda_{S}^{\prime}=1$. Once we have a collection of coalitions that is weakly balanced when only players with $w_{i}>0$ are considered, we can construct a collection in which the result is also true for players with $w_{i}=0$. Take a player $j$ with $w_{j}>0$, and add $i$ to the coalition if and only if $j$ is in it. Thus, coalitions including both $i$ and $j$ or neither are unchanged, coalitions including only $i$ have $i$ removed from them, and coalitions including only $j$ have $i$ added to them; the new coalitions inherit the weight of the old ones, and, since $w_{i}=0, i$ can be freely added or removed from coalitions in $W^{*}$ to obtain coalitions still in $W^{*}$. The resulting weights $\lambda_{S}^{\prime \prime}$ are such that $\sum_{S \in W^{*}, S \ni i} \lambda_{S}^{\prime \prime}=1$ for all $i$, hence $W^{*}$ is weakly balanced.

The nucleolus is always a solution to $(1)$, hence, when $W^{*}$ is weakly balanced, $w$ has the same maximum excess as the nucleolus. This does not imply that $w$ coincides with the nucleolus, or even that the nucleolus is a system of weights (see footnote 13 in the paper). Calculating the nucleolus is not a convenient way to solve (1): to calculate the nucleolus, one has to start by solving (1), which may have many solutions and, if this is the case, additional calculations have to be performed to determine which of the many solutions is the nucleolus. The upside of calculating the nucleolus is that researchers have developed algorithms and computer programs for this very purpose. Besides the more direct approach described in the supplementary files, all calculations 
in table 1 of the paper have been double-checked with the help of a computer program written by Jean Derks to compute the nucleolus.

\section{Proof of the corollary}

The proof of the corollary in the paper is very similar to the proof of the main proposition. For sufficiency, no changes need to be made since the proof does not rely on $w$ being a set of voting weights. The proof of necessity rests on the following lemma.

Lemma 2 Let $x$ be such that $x_{i}>0$ for all $i \in N$. If $v=x$ is a vector of equilibrium payoffs for the game with $\theta=x$, all players must belong to at least one of the cheapest winning coalitions in this equilibrium.

We have denoted the set of cheapest winning coalitions according to $x$ (the set of winning coalitions with minimum $\left.\sum_{i \in S} x_{i}\right)$ as $\mathcal{W}^{*}(x)$. Denote by $\bar{x}:=$ $\sum_{i \in S} x_{i}$ the total payoff of players in any such coalition (what we have denoted by $\bar{q}$ when $x$ is a set of weights). The proof of the analogous result in the main text relies on $\bar{q}>\frac{1}{2}$, which is known to hold since $w$ is a system of weights. The result holds more generally, but requires a longer proof.

Suppose an equilibrium exists with $v=x$. Consider the set $C$ of players that belong to at least one coalition in $\mathcal{W}^{*}(x)$. Since players only propose a coalition if it is among the cheapest winning coalitions to which they belong, players in $C$ only propose to other players in $C$, and they only propose coalitions of total payoff $\bar{x}$.

Take any coalition $S$ that $i \in C$ proposes with positive probability in equilibrium. The total expected payoff of players in $S$, including $i$, is $\sum_{j \in S} x_{j}=\bar{x}$ (the total actual payoff if $i$ is selected as a proposer and proposes $S$ is of course 
1). Player $i$ may play a mixed strategy as a proposer, but he always proposes a coalition of total expected payoff $\bar{x}$; hence, $\sum_{S: i \in S} p_{i}(S) \sum_{j \in S} x_{j}=\bar{x}$ in equilibrium, since each $S$ has a total expected payoff of $\bar{x}$ and $\sum_{S: i \in S} p_{i}(S)=1$.

We can re-arrange the expression $\sum_{S: i \in S} p_{i}(S) \sum_{j \in S} x_{j}$ to highlight the probabilities $p_{i j}$, where $p_{i j}$ is the probability that $i$ includes $j$ in the coalition (of course, $p_{i i}=1$ ). We then get

$$
\sum_{j \in C} p_{i j} x_{j}=\bar{x} \text { for all } i \in C .
$$

The next step is to look at $i$ 's expected payoff equation, where $i \in C$. We have $x_{i}=x_{i}\left(1-\delta\left(\bar{x}-x_{i}\right)\right)+r_{i} \delta x_{i}$, where we are already using $\theta=x$, and $r_{i}$ denotes the probability that $i$ is included in the coalition as a responder. Dividing by $x_{i}$, which we have assumed to be positive, we find $1=1-\delta(\bar{x}-$ $\left.x_{i}\right)+r_{i} \delta$, which we can re-arrange to find that all players in $C$ must be in the final coalition with probability $\bar{x}$.

Now suppose $N \backslash C$ is nonempty. We now show that this leads to a contradiction, hence $N=C$.

If $N \backslash C$ is nonempty, at least one player in $C$ must receive proposals from players in $N \backslash C$ since $C$ is a winning coalition and, given that the game is proper, this makes $N \backslash C$ a losing coalition. Thus, if we only consider proposals from players in $C$ to each other, we should find that at least one player in $C$ is in the coalition with a probability less than $\bar{x}$.

Taking expression (3), we can multiply both sides by $x_{i}$ to find $\sum_{j \in C} p_{i j} x_{j} x_{i}=$ $\bar{x} x_{i}$, and then add all such expressions up over $i$ to find

$$
\sum_{i \in C} \sum_{j \in C} p_{i j} x_{j} x_{i}=\bar{x} \sum_{i \in C} x_{i} .
$$

Now let us look at the left-hand side of (4). If we re-arrange the expression taking the point of view of the players $j$ who receive proposals, we have 
$\sum_{j \in C} \sum_{i \in C} p_{i j} x_{j} x_{i}=\sum_{j \in C} x_{j} \sum_{i \in C} p_{i j} x_{i}$. The expression $\sum_{i \in C} p_{i j} x_{i}$ is the probability that $j$ is included in the final coalition when only proposers from $C$ are considered; we know that this number is at most $\bar{x}$ for any $j$ and it is strictly below $\bar{x}$ for some $j$. Thus, $\sum_{j \in C} x_{j} \sum_{i \in C} p_{i j} x_{i}<\sum_{j \in C} x_{j} \bar{x}$, but this contradicts (4).

Given that $N=C$ and that $x_{i}>0$ for all $i$, since a player $i \in C$ with $x_{i}>0$ must be in the coalition with probability $\bar{x}$, the set $\mathcal{W}^{*}(x)$ must be weakly balanced. 